\title{
1 Component-Cost and Performance Based Comparison of Flow and Static
}

\section{Batteries}

3

4

${ }^{\mathrm{c}}$ Department of Mechanical Science and Engineering, University of Illinois at Urbana-Champaign, Urbana, Illinois 61801, USA

*Corresponding author’s email: ychiang@mit.edu

${ }^{\mathrm{a} D}$ Department of Mechanical Engineering, ${ }^{\mathrm{b}}$ Department of Material Science and Engineering, Massachusetts Institute of Technology, Cambridge, Massachusetts 02139, USA 


\section{Abstract}

Flow batteries are a promising grid-storage technology that is scalable, inherently flexible in power/energy ratio, and potentially low cost in comparison to conventional or "static" battery architectures. Recent advances in flow chemistries are enabling significantly higher energy density flow electrodes. When the same battery chemistry can arguably be used in either a flow or static electrode design, the relative merits of either design choice become of interest. Here, we analyze the costs of the electrochemically active stack for both architectures under the constraint of constant energy efficiency and charge and discharge rates, using as case studies the aqueous vanadium-redox chemistry, widely used in conventional flow batteries, and aqueous lithium-iron-phosphate (LFP)/lithium-titanium-phosphate (LTP) suspensions, an example of a higher energy density suspension-based electrode. It is found that although flow batteries always have a cost advantage $\left(\$ \mathrm{kWh}^{-1}\right)$ at the stack level modeled, the advantage is a strong function of flow electrode energy density. For the LFP/LTP case, the cost advantages decreases from 50\% to $\sim 10 \%$ over experimentally reasonable ranges of suspension loading. Such results are important input for design choices when both battery architectures are viable options.

\section{Keywords}

flow battery, cost model, vanadium, lithium iron phosphate, lithium titanium phosphate, electrochemistry 


\section{Introduction}

Low-cost electrical energy storage can greatly facilitate the integration of intermittent renewable power generation into the electric grid [1]. However, the cost of available energy storage technologies has been too high for most large-scale grid applications [2,3]. Flow batteries are considered to be promising candidates for large-scale storage due to their inherent scalability and decoupled power and energy. The cost per stored energy, e.g., $\$ \mathrm{kWh}^{-1}$, of flow batteries generally decreases as the ratio of tank size to reactor size increases. However, simultaneously the power capability and round trip energy efficiency also decrease, all else being equal. Thus one may ask, "For any given chemistry, when is it cost effective to use a flow battery architecture as opposed to a conventional static-electrode architecture?" Cost analyses exist for both static [4-6] and flow [7-10] batteries independently, but we are not aware of published works that compare the cost merits of the two architectures for the same active materials and performance requirements.

The question has increased in relevance with the emergence of flowable storage electrodes of high energy density. Historically, flow batteries have used dissolved redox molecules as electroactive materials [1]. Recently, however, alternative forms of flow electrodes including semi-solid suspensions of the electroactive materials typically used in nonaqueous Li-ion batteries [4] and conductive suspensions of high energy density solution chemistries such as lithium-polysulfide $[11,12]$ have been reported. These electrodes have the potential to increase the chemical energy density by an order of magnitude or more over aqueous redox solutions. 
Evaluation of the performance/cost trade-off of different battery architectures is especially important in these instances.

We first answer the question of "when to build a flow battery" conceptually, and then support our conclusions with analysis and case studies in the remainder of the paper. A flow battery architecture is in general more cost effective than a static battery architecture when chemical cost is low relative to the cost of the separator membrane and current collector, and when the anode and cathode solutions or suspensions have low volumetric energy densities. As energy density of reactants increases, a flow battery design can remain more attractive from a cost perspective, but the benefit over a static electrode architecture diminishes. A critical consideration in arriving at this conclusion is the fact that for any given electrode chemistry used at a given C-rate of charge or discharge in a planar configuration, the polarization due to electrode resistance increases in proportion to the electrode thickness squared. This dependence, simple yet not widely appreciated, arises because polarization is the product of current density and electrode resistance (i.e., Ohm's law); the resistance of a uniform electrode scales linearly with its thickness, but so does the current density needed to maintain a given C-rate.

Battery costs can be decreased by decreasing the inactive material content, e.g., the number of separator and current collector layers, and for a layered design this inactive content should be

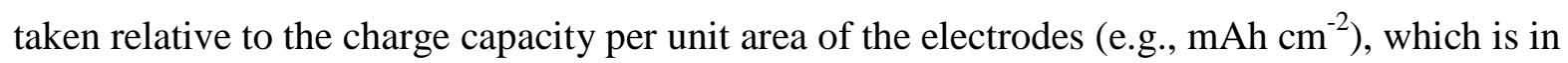
turn proportional to thickness for a given electrode composition. Thus, while low energy density electrodes must be thick in order to minimize the inactive material content, increasing electrode 
thickness incurs a penalty in polarization that limits usable capacity (i.e., that is accessible within the voltage window of the cell) and round-trip efficiency.

For these reasons, our model assumes a constraint of identical round-trip efficiency when comparing flow and static-electrode architectures. The analysis presented below considers the vanadium redox solution as the exemplar of an aqueous flow battery, and an aqueous suspension-based lithium-iron-phosphate (LFP)/lithium-titanium-phosphate (LTP) electrochemical couple, recently demonstrated in both static and flow battery configurations [15], as an example of a higher energy density chemistry, which was achieved primarily using compounds of higher charge capacity per unit volume. Given the expanding number of electroactive material options that can be used in both static-electrode and flow batteries, our purpose is to present analysis methods that can be incorporated into design tools for evaluating the economics and performance/cost trade-offs of battery designs.

\section{Cost Model}

A cost model for either flow or conventional batteries would ideally take into account the cost of all components (bill of materials), manufacturing capital expenditures (depreciated over lifetime), and labor/operating costs. We seek to avoid such specificity in the present work since the results are specific to particular designs (e.g., energy and duration for flow batteries; cell size and power for conventional batteries) and can obscure the fundamental relationships that will be elucidated. Instead, we seek to compare flow and static-electrode batteries in terms of an irreducible subsystem shown in Fig. 1 that consists of the cell "stack" in the case of a conventional laminated battery and its equivalent in the case of the flow battery. This subsystem 
contains all components of the electrochemically active electrodes-cathode, anode, electrolyte, and additives - and the separator films and cathode and anode current collectors. Thus for the static-electrode cell, we model the cost of the stack and exclude the cost of packaging, tabs, and any control electronics. For the flow battery, the model includes the total "chemical cost" and the major components of the power-generating reactor and excludes the balance of the plant.

In our model, we require that both the flow battery and static-electrode battery subsystems as defined above $(i)$ have identical round-trip energy efficiency at specified charge and discharge rates, (ii) use the same components for the current collector, separator, and solutions or suspensions, and therefore have the same component unit costs, and (iii) consist of the same formulations, and therefore energy densities, for the chemically active electrodes. The theoretical capacity and energy of the system is the same for both batteries (Fig. 1). The staticelectrode battery will therefore have thicker electrodes and more separator and current collector per unit of stored energy. The flow battery will utilize a thinner, higher power stack through which the catholyte and anolyte must be flowed at the necessary rate to achieve the same systemlevel C-rate or power as the static-electrode battery.

The stack cost per kilowatt hour of a static or flow battery, $C_{s t}$, under these assumptions is the total cost of the solution or suspension, $C_{a c}$, separator membrane, $C_{s}$, and current collector, $C_{c}$, divided by the total amount of usable energy storage, $E$, or $C_{s t}=\left(C_{a c}+C_{s}+C_{c}\right) / E$. The total costs are calculated as follows, $C_{a c}=\left(P c_{a c}\right) /\left(C \rho_{e}\right), C_{m}=A_{s} c_{s}, C_{c}=A_{c} c_{c}$, and $E=\left(P \eta_{e}\right) / C$. The variables $c_{a c}, c_{s}$, and $c_{c}$ are the average cost of anode and cathode per unit mass, the separator cost per unit area, and the current collector cost per unit area respectively. $P$ is the average 
charging and discharging power. $C$ is the system-level C-rate at which the battery charges/discharges. The theoretical gravimetric energy density is $\rho_{e}$, and $\eta_{e}$ is the energetic efficiency. The variables $A_{c}$ and $A_{s}$ represent the total area of current collector and separator respectively and are related as $A_{c}=A_{s}(1+1 / n)$, assuming current collectors are shared by adjacent cells and where $n$ is the number of cells in the battery.

Flow batteries can use less separator and current collector than static batteries for the same energy storage, which is why their cost per kilowatt-hour decreases as their tank size increases. The trade-off, however, is that the C-rate of the flow battery stack must be higher, by a factor equal to the ratio of tank to stack capacity, to achieve a net $\mathrm{C}$-rate equal to that of a comparable static battery. To achieve the same energy efficiency as the static battery, the thinner electrode thickness is a benefit, but the higher C-rate is a detriment. Our model takes both into account as well as the pumping losses for the stack. We use the following terminology. The volume or charge capacity that the stack alone can accommodate is referred to as a plug. The C-rate of an individual plug is defined as $C_{p}$. The number of plugs in the system, or plug count, $p$, is the total anode or cathode capacity in the system, taken as a multiple of that for the stack. A static battery has a plug count of one, and a flow battery has a plug count greater than one. Figure $1 \mathrm{~b}$ illustrates a flow battery with a plug count of four. The average charging and discharging power of the battery, static or flowing, can be approximated as $P=2 h_{p} l w \rho \rho_{e} C_{p} n$. Electrode thickness (distance from membrane to current collector), $h_{p}$, is varied to achieve a particular energy efficiency for a specified system-level C-rate and plug count $p$. The variables $l$ and $w$ are dimensions of the area of separator required for a single cell as shown in Fig. 2, and $\rho$ is the average of the gravimetric densities of the anode and cathode solutions or suspensions. If the 
total separator area $A_{m}$, which is equal to $l w n$, is divided by both sides of the approximated power expression, the total amount of required separator is $A_{m}=P /\left(2 h_{p} \rho \rho_{e} C_{p}\right)$. These expressions can be substituted into the cost per kilowatt-hour expression to obtain the cost of the stack relative to system energy $\left(\$ \mathrm{kWh}^{-1}\right)$ :

$$
C_{s t}=\frac{1}{\eta_{e} \rho_{e}}\left(c_{a c}+\frac{c_{s}+c_{c}\left(1+\frac{1}{n}\right)}{2 h_{p} \rho p}\right)
$$

$C_{s t}$ is the stack energy cost of a static battery when $p=1$ and that of a flow battery when $p>1$. The difference in stack cost between a static and a flow battery, $C_{s-f}$, is:

$$
C_{s-f}=\frac{c_{s}+c_{c}\left(1+\frac{1}{n}\right)}{2 \eta_{e} \rho \rho_{e}}\left(\frac{1}{h_{1}}-\frac{1}{h_{p} p}\right)
$$

Equation 2 shows that the difference in stack cost between static and flow batteries is maximized when (i) anode and cathode solutions or suspensions have low volumetric densities and low energy densities, and (ii) the cost of separator and current collector is high. The dependence of cost on electrode thicknesses, $h_{1}$ for the static cell and $h_{p}$ for the flow cell, is less obvious and requires consideration of the dependence of charge and discharge rate and efficiency on thickness as discussed in the next section.

\section{Energy-Efficiency Model}


Energy efficiency, the ratio of discharge energy to charge energy, is calculated taking into account both electrochemical losses and pumping losses, the latter applying only to the flow battery. In the case of electrochemical efficiency, we assume that the same coulombic efficiency is obtained for a given electrochemical couple in both battery architectures such that the energy efficiency is determined by voltage inefficiency alone. We later incorporate the inefficiency due to pumping. The energy efficiency $\eta_{\mathrm{e}}$ therefore depends on the time-averaged polarization, $\Delta \Phi$, and the average open-circuit potential, $\bar{\phi}_{e q}$, of the anode and cathode pair.

$\eta_{e}=\frac{\bar{\phi}_{e q}-\Delta \Phi}{\bar{\phi}_{e q}+\Delta \Phi}$

Time-averaged polarization was modeled by assuming that (i) surface-reaction kinetics and porescale mass-transfer of ionic and redox-active species are facile processes (an assumption that we later relax for the V-redox cell), (ii) electrochemical reactions in either electrode are focused at a propagating front, and (iii) both electronic and ionic ohmic processes dominate polarization. Implicitly, this model neglects efficiency losses due to flow-profile non-uniformity [13], shunt currents, extension of the electroactive zone [14], and depletion of dissolved species.

Under these assumptions, the time-averaged polarization is proportional to area-specific resistance, $R_{a s r}$, and current density $I$, i.e. $\Delta \Phi=i R_{a s r}$, where current density is the product of the cell's area-specific capacity and plug-level C-rate. Area-specific resistance includes contributions from the anode (subscript $a$ ), cathode (subscript $c$ ), and separator (subscript $s$ ) 
shown in Eq. 4 where $h_{j}, \sigma_{e f f ;}$, and $\kappa_{e f f ; j}$ are respectively the electrode thickness, effective electronic conductivity, and effective ionic conductivity of electrode $j$.

$$
R_{a s r}=\left[\frac{h_{a}}{2}\left(\frac{1}{\sigma_{e f f, a}}+\frac{1}{\kappa_{e f f, a}}\right)+R_{s}+\frac{h_{c}}{2}\left(\frac{1}{\sigma_{e f f, c}}+\frac{1}{\kappa_{e f f, c}}\right)\right]
$$

When vanadium-redox solutions are flowed through porous carbon current collectors, surface reaction kinetics can produce substantial polarization that is not accounted for in Eq. 4. We account for this polarization by also including in $\Delta \Phi$ the kinetic overpotentials produced by uniform reactions in both electrodes. Uniform reactions occur strictly at low current densities where transport is kinetically limited, but here we use this result to estimate kinetic effects at all current densities. Using the Butler-Volmer equation with equal anodic and cathodic transfer coefficients, the overpotential $\eta_{j}$ for electrode $j$ is expressed in Eq. 5.

$$
\eta_{j}=\frac{2 R_{g} T}{F} \operatorname{asinh}\left(\frac{i}{2 h_{j} i_{0, j} a_{s, j}}\right)
$$

Here, the exchange current density and electroactive surface-area per unit electrode-volume are $i_{0, j}$ and $a_{s, j}$, respectively, and $R_{g}, T$, and $F$ are the universal gas constant, temperature, and Faraday's constant.

The flow battery has additional losses due to pumping the fluid through the stack channels. Due to the need for thin electrodes to maximize electrochemical efficiency, the pumping losses are likely much greater in the stack than anywhere else in the system. The pumping losses are a 
function of the fluid properties of the electrode, the electrode thickness, and the length of the flow channel, and are calculated as follows. Flow channels of length $l$ with a high aspect ratio rectangular cross-section $\left(w>>h_{p}\right)$ are assumed, as this cross-section minimizes pumping losses. The pumping losses are calculated as a percentage of the theoretical energy density of the system by assuming that full depth-of-discharge is achieved in a single flow-pass, i.e., stoichiometric flow conditions are used. The energy density per unit volume dissipated to pump suspension or solution through the cell in a single pass is calculated as $\Delta P(1-1 / p) . \Delta P$ is the pressure-drop across the static cell's current collector, and its calculation depends on the type of fluid employed in the flow cell, as described subsequently. Solution and suspension pressure-drops were calculated according to the inset equations in Figs. S1a and S1b, respectively. For the suspension, plastic viscosity is neglected, because for the cases shown, the elastic stresses dominate the flow, which is reflected by a Bingham number, the characteristic ratio of elastic-toviscous flow stresses, that is greater than unity [13].

The total energy efficiency including both electrochemical and mechanical losses is constrained to be $95 \%$ in most of the results shown. Channel lengths $l$ of $100 \mathrm{~mm}$ to $3 \mathrm{~m}$ are evaluated, as this encompasses a realistic range of stack dimensions. The pressure drop $\Delta P$ is calculated as a function of plug count $p$ for cells having (a) vanadium-redox solution and (b) suspension with 10 vol $\%$ LFP. For each plug count, $\Delta P$ was calculated for the thickness $h_{p}$ corresponding to the specified energy efficiency including electrochemical and mechanical energy-losses at $\mathrm{C} / 5$ system-level rate and with separator length $l$. Continuous, stoichiometric flow conditions were assumed. The pressure drop results are shown in Fig. S1 in the Supplemental Information to this article. 
The LFP suspension was assumed to have a Bingham plastic rheology with yield shear-stress $\tau_{0}$ equal to $6.5 \mathrm{~Pa}$, as measured previously for 10 vol\% LFP with 1 vol\% Ketjen black [15].

250

Suspensions with 20 vol\% and 40 vol\% active material were modeled with yield shear-stresses of $62 \mathrm{~Pa}$ and $172 \mathrm{~Pa}$, respectively. These values were estimated by linear extrapolation of yield shear-stress data in Ref. [15] with LFP loading for suspensions with 1vol\% Ketjen black.

Solution pressure-drop caused by flow through a porous current collector was modeled using the inset equation in Fig. S1a assuming Darcy flow for a saturated $\mathrm{V}^{\mathrm{IV}}$ solution in $5 \mathrm{~mol} \mathrm{~L}^{-1} \mathrm{H}_{2} \mathrm{SO}_{4}$ with a viscosity of $10 \mathrm{mPa} \mathrm{s}[16]$ and a hydraulic permeability $k$ of $10^{-11} \mathrm{~m}^{2}$ for $7 \mu \mathrm{m}$ diameter carbon-fiber felt at $20 \mathrm{vol} \%$ [17]. The electrode thickness that gives 95\% round trip energy efficiency (or other values noted later), taking into account both electrochemical and pumping losses, was then used as input to determine the relative costs of the flow and static cells.

\section{Properties of Model Chemistries}

The average open-circuit potential is taken as $1.3 \mathrm{~V}$ for the vanadium-redox chemistry [18] and $0.9 \mathrm{~V}$ for the LFP/LTP chemistry [15]. For the $2 \mathrm{~mol} \mathrm{~L}^{-1}$ vanadium-redox solution, a volumetric capacity of $53.6 \mathrm{mAh} \mathrm{cm}^{-3}$ is assumed. For the LFP/LTP chemistry, it is assumed that the cell is cathode-limited with a theoretical capacity of $610 \mathrm{mAh} \mathrm{cm}^{-3}$ based on LFP volume only [19]. The effective electronic conductivity used for both electrodes of the vanadium-redox cell is 100 $\mathrm{S} \mathrm{m}^{-1}$, estimated for a flow-through carbon felt. The electronic conductivity of both electrodes of the LFP/LTP cell is assumed to be $0.1 \mathrm{~S} \mathrm{~m}^{-1}$, consistent with an aqueous semi-solid suspension that uses a percolating network of nanoscale carbon [15]. The vanadium-redox cell is assumed to have an ionic conductivity for both electrodes of $600 \mathrm{mS} \mathrm{cm}^{-1}$ as expected for $5 \mathrm{~mol} \mathrm{~L}^{-1} \mathrm{H}_{2} \mathrm{SO}_{4}$ 
supporting electrolyte [20]. The electrolyte phase of the LFP and LTP suspensions is taken to be an aqueous solution of $1 \mathrm{~mol} \mathrm{~L}^{-1} \mathrm{LiNO}_{3}$, which at $300 \mathrm{~K}$ has an ionic conductivity of $7 \mathrm{~S} \mathrm{~cm}^{-1}$ [21]. Effective ionic conductivities for the particle-loaded suspensions are calculated from the Bruggeman approximation (i.e., $\kappa_{e f f}=\kappa_{o} \varepsilon^{1.5}$, where $\varepsilon$ and $\kappa_{o}$ are porosity and bulk ionic conductivity, respectively) assuming porosity is occluded only by the active-material particles. For the area-specific resistance of the ion-selective separator in the vanadium-redox cell, we use a measured value for Nafion 117 of $R_{s}=200 \mathrm{~m} \Omega-\mathrm{cm}^{2}$ [22]. For the LFP/LTP cell, the areaspecific resistance of the separator depends on its effective ionic conductivity, $\kappa_{\text {eff,s, }}$, and thickness, $h_{s}$, as $R_{s}=h_{s} / k_{\text {eff,s } s}$. For a $50 \%$ porous, $25 \mu \mathrm{m}$ separator in aqueous electrolyte, this produces an area-specific resistance of $100 \mathrm{~m} \Omega \mathrm{cm}^{2}$. Exchange current densities for the cathodic and anodic vanadium-redox reactions were measured in situ by Aaron et al. [23]. They tested solutions having $0.1 \mathrm{~mol} \mathrm{~L}^{-1}$ vanadium, and therefore we scale their reported values of exchangecurrent density for the $2.0 \mathrm{~mol} \mathrm{~L}^{-1}$ solutions investigated here assuming first-order dependence on vanadium concentration [24] $i_{0, a}=0.045 \mathrm{~mA} \mathrm{~cm}^{-2}$ and $i_{0, c}=2 \mathrm{~mA} \mathrm{~cm}^{-2}$. We use an electroactive surface area for both electrodes of $1.6 \times 10^{5} \mathrm{~m}^{-1}$ for SGL 10 AA carbon paper [25], which was used by Aaron et al. [23].

The assumed costs of components are as follows. The costs of carbon black, $\mathrm{H}_{2} \mathrm{O}$, and $\mathrm{H}_{2} \mathrm{SO}_{4}$ are negligible compared to other components. The costs of vanadium, LTP, and LFP are assumed to be $21 \$ \mathrm{~kg}^{-1}$ [7], $15 \$ \mathrm{~kg}^{-1}$, and $15 \$ \mathrm{~kg}^{-1}$, respectively [26]. For the vanadium redox battery, the cost of the current collector and carbon felt combined is assumed to be $71 \$ \mathrm{~m}^{-2}$ [7] and the membrane cost is assumed to be $500 \$ \mathrm{~m}^{-2}$ [7]. For the LFP/LTP cell, the costs of the 
current collector and separator are assumed to be $0.90 \$ \mathrm{~m}^{-2}$ and $2 \$ \mathrm{~m}^{-2}$, respectively, [26] assuming the aluminum current collector thickness is $15 \mu \mathrm{m}$.

\section{Results and Discussion}

The cost and energy-efficiency models were combined to calculate the stack cost as a function of plug count of the outlined vanadium redox flow battery and aqueous suspension-based LFP/LTP battery assuming a fixed energetic efficiency of $95 \%$ and a system-level charge and discharge rate of $\mathrm{C} / 5$. Figure 3 shows the pumping losses for the vanadium redox solution (Fig. 3a) and LFP/LTP suspensions (Fig. 3b), respectively, for plug counts ranging from one, i.e., the static cell, to 30 . Note firstly that the pumping losses are lower than the electrochemical losses for channel lengths $<1 \mathrm{~m}$. Secondly, the suspension has lower pumping losses than the solution throughout. This is perhaps counterintuitive given that the suspensions have higher viscosity and are yield-stress fluids. The lower pumping losses are due to the fact that the conductive suspensions, having an embedded current collector, do not require a separate porous carbon current collector but instead can use an unobstructed flow channel. The use of percolating nanoconductor networks as embedded current collectors was described in detail in reference [12] for a conductive suspension-based lithium polysulfide flow batteries. In the plots in Fig. 3, the electrode thickness varies continuously along each of the curves in order to maintain constant 95\% energy efficiency. The corresponding electrode thicknesses are shown in Fig. 4, now calculated for the full cells. Here, the electrode thickness is nearly independent of channel length for the LFP/LTP suspensions (Fig. 4b) because the electrochemical losses dominate. For the vanadium redox solution (Fig. 4a), this is also true for $l<1 \mathrm{~m}$, but above $1 \mathrm{~m}$ the pumping losses 
increase such that at $l=3 \mathrm{~m}$, for example, the electrode thickness required to maintain $95 \%$ energy efficiency drops off quickly to zero with increasing plug count. This observation clearly suggests that the vanadium redox solution has difficulty meeting the $95 \%$ efficiency requirement at $\mathrm{C} / 5$ rate using realistic cell design parameters, as discussed further below. In Figs. $4 \mathrm{a}$ and $4 \mathrm{~b}$, the value of the electrode thickness at a plug count of unity (i.e., zero pumping loss) is the thickness of an electrode that yields 5\% electrochemical loss.

Taking the electrode thicknesses in Fig. 4, the corresponding stack cost vs. plug count are directly computed from Eq. 1, and the cost differential between flow and static cells is calculated from Eq. 2. The results are plotted in Figs. 5 and 6 respectively. The cost of chemical constituents only for $95 \%$ energetic efficiency, which the flow cell cost must asymptotically approach as plug count increases, is shown in Fig. 5 as a horizontal line. Note that costs for the vanadium redox solution, Fig. 5a, exhibit a minimum at plug count $<5$, with a relatively high cost of $\sim 300-400 \$ \mathrm{kWh}^{-1}$. This is because meeting $95 \%$ energy efficiency at $\mathrm{C} / 5$ rate requires large stacks with thin electrodes, which drives up cost. However, if the energy efficiency is relaxed to $85 \%$, Fig. $5 \mathrm{a}$, a broader minimum with cost as low as $\sim 150 \$ \mathrm{kWh}^{-1}$ is achievable. In contrast to this behavior, the Li-ion suspension chemistry, Fig. 5b, has inherently higher stack efficiency and lower pumping losses, and the cost continues to decrease towards the chemical cost limit (horizontal line) with increasing plug count.

As stated at the outset, one expects that as the energy density of the electrode increases, the cost benefit of the flow architecture over the static battery should diminish. With the above results in hand, we can evaluate this behavior quantitatively for the suspension case (the trends are 
qualitatively similar for the vanadium redox case). The results above assumed a 10 vol\% LFP suspension, for which the rheological behavior has been characterized [15]. Although the rheology at lower and higher loadings has not been measured, by linearizing existing rheology data [15], we can approximate the pumping losses for such scenarios. We calculate in Fig. 6a the stack cost vs. plug count for 1 vol\%, 10 vol\%, 20 vol\% and 40 vol\% LFP, again imposing the requirement of $95 \%$ efficiency at $\mathrm{C} / 5$ rate. It is seen that increasing energy density (vol\% LFP) enables lower cost and lower plug counts. Thus within the flow battery architecture, increasing energy density clearly lowers cost for the same performance.

In Fig. $6 b$ the cost differential between static and flow cells is shown as a percentage of the static cell cost. Although the flow cell "stack" always has a cost advantage over the static cell, this advantage is $\sim 50 \%$ at the lowest concentrations but diminishes to $<10 \%$ at 40 vol\% LFP (corresponding to a molar concentration of $9.1 \mathrm{M}$ ) for any reasonable plug count.

The non-stack costs of both the static and flow battery may of course be a substantial percentage of total cost and would need to be treated in detail to obtain a more refined comparison. Other component costs, manufacturing costs, and any differences in manufacturing yield would need to be included. Taking an example where the non-stack costs are 50\% for both architectures, Fig. $6 \mathrm{~b}$ indicates that the cost advantage of the flow cell may be only $10 \%$ at 40 vol\% $(9.1 \mathrm{M})$, but increases to $\sim 50 \%$ at $1 \mathrm{vol} \%(0.23 \mathrm{M})$. These numerical results support the conclusion that flow cell architectures are most favorable when used with low energy density catholytes and anolytes such as aqueous redox solutions that have to date dominated flow batteries. However, as the energy density of flow electrodes increases, the cost-competitiveness of static cell designs 
increases, and detailed consideration of tradeoffs in complexity and manufacturing are necessary to evaluate the merits of the competing designs.

\section{Conclusions}

The cost of energy storage using flow batteries vs. conventional or "static" batteries has been modeled at an electrochemical subsystem level that includes the cost of chemistry and stack components, but excludes packaging, hardware, and balance of plant. Cost comparisons are carried out for the same chemistries at the same round trip energy efficiency of $95 \%$ at $\mathrm{C} / 5$ rate (for the entire system) wherein the round trip efficiency takes into account electrochemical and stack pumping losses. Two electrochemical couples have been selected for which sufficient data exists to model the electrochemical efficiency as a function of cell design parameters: vanadium redox solutions and a model suspension-based Li-ion flow chemistry using LFP/LTP. It is found that although flow batteries always have a $\$ \mathrm{kWh}^{-1}$ cost advantage at this subsystem level, the advantage is a strong function of electrolyte energy density. For the LFP/LTP case, the cost advantages decreases from $\sim 50 \%$ to $\sim 10 \%$ over experimentally reasonable ranges of suspension loading, thereby informing design choices when both battery architectures are viable options.

\section{Acknowledgements}

This work was supported as part of the Joint Center for Energy Storage Research, an Energy Innovation Hub funded by the U.S. Department of Energy, Office of Science, Basic Energy Sciences. BJH acknowledges helpful discussions with Victor Brunini on flow-battery costanalysis. 


\section{List of Acronyms}

LFP Lithium Iron phosphate

390

LTP Lithium Titanium phosphate

391

392

\section{Nomenclature (units)}

$393 C_{s t} \quad$ stack cost per unit energy of static or flow battery $\left(\$ \mathrm{kWh}^{-1}\right)$

$394 \rho \quad$ average density of anode and cathode material $\left(\mathrm{kg} \mathrm{m}^{-3}\right)$

$395 \rho_{e} \quad$ theoretical gravimetric energy density $\left(\mathrm{kWh} \mathrm{kg}^{-1}\right)$

$396 \quad C \quad$ C-rate at which battery charges/discharges $\left(\mathrm{h}^{-1}\right)$

$397 \quad C_{p} \quad$ C-rate of plug $\left(\mathrm{h}^{-1}\right)$

$398 \quad p \quad$ plug count (-)

$399 E \quad$ total usable energy $(\mathrm{kWh})$

$400 \quad P \quad$ average charging/discharging power $(\mathrm{kW})$

$401 \eta_{e} \quad$ energetic efficiency (-)

$402 A_{c} \quad$ total area of current collector $\left(\mathrm{m}^{2}\right)$

$403 A_{s} \quad$ total area of separator $\left(\mathrm{m}^{2}\right)$

$404 C_{a c} \quad$ total cost of anode and cathode materials (\$)

$405 \quad C_{c} \quad$ total cost of current collector (\$)

$406 C_{s} \quad$ total cost of separator (\$) 
$407 \quad c_{a c} \quad$ average cost of anode and cathode current collectors per unit mass $(\$ \mathrm{~kg})$

$408 \quad c_{s} \quad$ separator cost per unit area $\left(\$ \mathrm{~m}^{-2}\right)$

$409 \quad c_{c} \quad$ current collector cost per unit area $\left(\$ \mathrm{~m}^{-2}\right)$

$410 \quad n \quad$ number of cells in battery (-)

$411 \quad l \quad$ length of separator in single cell (m)

$412 \quad w \quad$ width of separator in single cell (m)

$413 \quad h_{p} \quad$ electrode thickness or distance from separator to current collector (m)

$414 \Delta \Phi \quad$ time-averaged polarization $(\mathrm{V})$

$415 \bar{\phi}_{e q} \quad$ average open-circuit potential (V)

$416 \quad R_{a s r} \quad$ area-specific resistance $\left(\Omega \mathrm{m}^{2}\right)$

$417 \quad i \quad$ current density $\left(\mathrm{A} \mathrm{m}^{-2}\right)$

$418 \quad h \quad$ thickness of electrode $(\mathrm{m})$

$419 \sigma_{e f f} \quad$ effective electronic conductivity of electrode $\left(\mathrm{S} \mathrm{m}^{-1}\right)$

$420 \kappa_{e f f}$ effective ionic conductivity of electrode $\left(\mathrm{S} \mathrm{m}^{-1}\right)$

$421 \quad \varepsilon \quad$ porosity (-)

$422 \quad \kappa_{o} \quad$ bulk ionic conductivity $\left(\mathrm{S} \mathrm{m}^{-1}\right)$

$423 \quad R_{s} \quad$ area-specific resistance of the ion-selective separator in the vanadium-redox cell $\left(\Omega \mathrm{m}^{2}\right)$

$424 \quad \eta \quad$ overpotential (V)

$425 \quad R_{g} \quad$ universal gas constant $\left(\mathrm{J} \mathrm{K}^{-1} \mathrm{~mol}^{-1}\right)$

$426 \quad T \quad$ temperature $(\mathrm{K})$

$427 \quad F \quad$ Faraday's constant $\left(\mathrm{C} \mathrm{mol}^{-1}\right)$

$428 \quad a \quad$ electroactive surface-area per unit electrode-volume $\left(\mathrm{m}^{-1}\right)$ 


\section{References}

[1] B. Dunn, H. Kamath, J.-M. Tarascon, Science 334 (2011) 928.

[2] S.M. Schoenung, W. V Hassenzahl, Long- vs . Short-Term Energy Storage Technologies Analysis: A Life-Cycle Cost Study: A Study for the DOE Energy Storage Systems Program, Albuquerque, New Mexico, 2003.

[3] “RECOVERY ACT” FINANCIAL ASSISTANCE FUNDING OPPORTUNITY ANNOUNCEMENT U. S. Department of Energy - Headquarters Advanced Research Projects Agency - Energy ( ARPA-E ) Grid-Scale Rampable Intermittent Dispatchable Storage ( GRIDS ) DE-FOA-0000290, 2010.

[4] P.A. Nelson, K.G. Gallagher, I. Bloom, D.W. Dees, Modeling the Performance and Cost of Lithium-Ion Batteries for Electric-Drive Vehicles, Argonne, 2012.

[5] M. a Delucchi, T.E. Lipman, Transp. Res. Part D Transp. Environ. 6 (2001) 371.

[6] D.N. Rakhmatov, S.B.K. Vrudhula, IEEE/ACM Int. Conf. Comput. Aided Des. ICCAD 2001. IEEE/ACM Dig. Tech. Pap. (Cat. No.01CH37281) (2001) 488.

[7] M. Zhang, M. Moore, J.S. Watson, T. a. Zawodzinski, R.M. Counce, J. Electrochem. Soc. 159 (2012) A1183.

[8] D.P. Scamman, G.W. Reade, E.P.L. Roberts, J. Power Sources 189 (2009) 1231.

[9] R.M. Darling, K.G. Gallagher, J. a. Kowalski, S. Ha, F.R. Brushett, Energy Environ. Sci. 7 (2014) 3459.

[10] V. Viswanathan, A. Crawford, D. Stephenson, S. Kim, W. Wang, B. Li, G. Coffey, E. Thomsen, G. Graff, P. Balducci, M. Kintner-Meyer, V. Sprenkle, J. Power Sources 247 (2014) 1040.

[11] Y. Yang, G. Zheng, Y. Cui, Energy Environ. Sci. 6 (2013) 1552.

[12] F.Y. Fan, W.H. Woodford, Z. Li, N. Baram, K.C. Smith, A. Helal, G.H. McKinley, W.C. Carter, Y. Chiang, Nano Lett. 14 (2014) 2210. 
[13] K.C. Smith, Y.-M. Chiang, W.C. Carter, J. Electrochem. Soc. 161 (2014) A486.

[14] K.C. Smith, V.E. Brunini, Y. Dong, Y.-M. Chiang, W.C. Carter, Rev. (n.d.).

[15] Z. Li, K.C. Smith, Y. Dong, N. Baram, F.Y. Fan, J. Xie, P. Limthongkul, W.C. Carter, Y.M. Chiang, Phys. Chem. Chem. Phys. 15 (2013) 15833.

[16] G. Oriji, Y. Katayama, T. Miura, Electrochim. Acta 49 (2004) 3091.

[17] A.Z. Weber, M.M. Mench, J.P. Meyers, P.N. Ross, J.T. Gostick, Q. Liu, J. Appl. Electrochem. 41 (2011) 1137.

[18] M. Skyllas-Kazacos, M. Rychcik, R.G. Robins, A.G. Fane, M.A. Green, J. Electrochem. Soc. 133 (1986) 1057.

[19] A.K. Padhi, K.S. Nanjundaswamy, J.B. Goodenough, J. Electrochem. Soc. 144 (1997).

[20] F. Rahman, M. Skyllas-Kazacos, J. Power Sources 189 (2009) 1212.

[21] A.N. Campbell, G.H. Debus, E.M. Kartzmark, Can. J. Chem. 33 (1955) 1508.

[22] F.N. Büchi, G.G. Scherer, J. Electroanal. Chem. 404 (1996) 37.

[23] D. Aaron, C.-N. Sun, M. Bright, a. B. Papandrew, M.M. Mench, T.A. Zawodzinski, ECS Electrochem. Lett. 2 (2013) A29.

[24] R.M. Darling, M.L. Perry, J. Electrochem. Soc. 161 (2014) A1381.

[25] S.C. Barton, Y. Sun, B. Chandra, S. White, J. Hone, Electrocheimcal Solid-State Lett. 10 (2007) B96.

[26] W.F. Howard, R.M. Spotnitz, J. Power Sources 165 (2007) 887. 
Figures
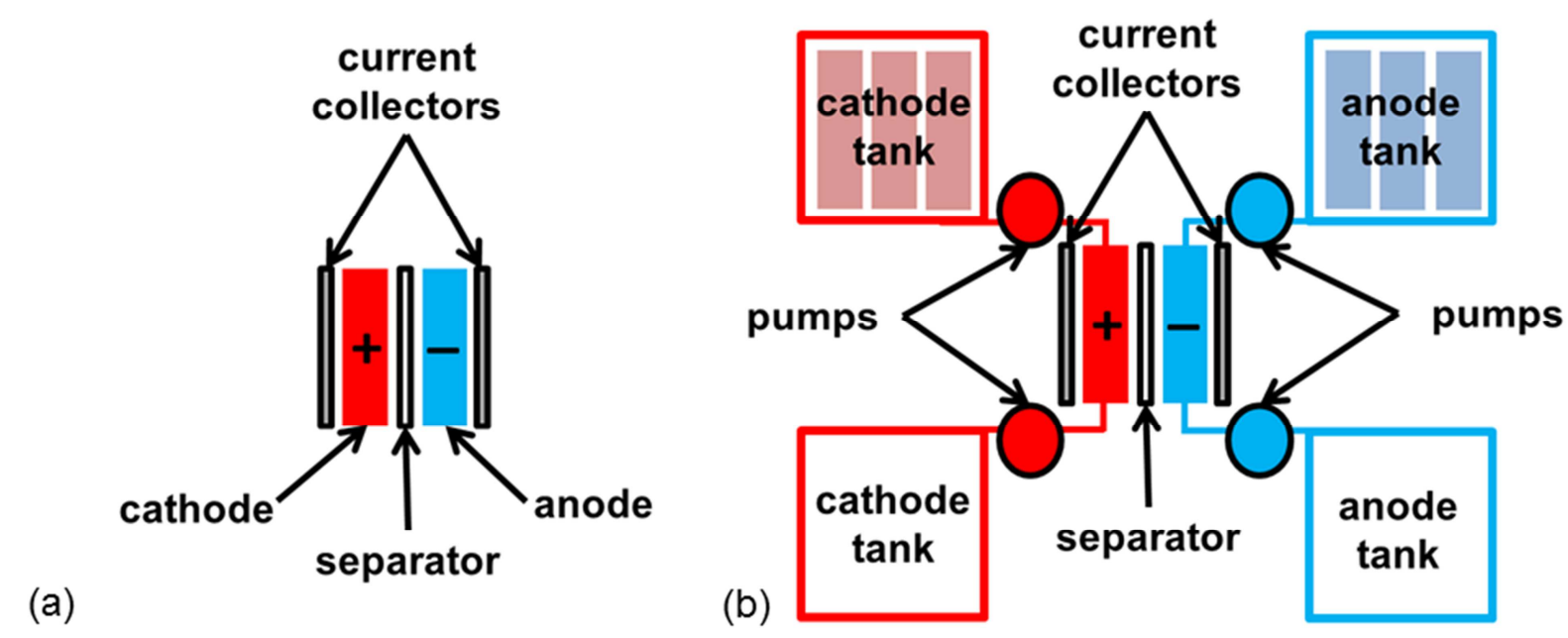

Fig. 1. (a) Static battery with plug count of one. (b) Flow battery with plug count equal to four if the contribution of solutions or suspensions stored in the piping and pumps is considered to be negligible.

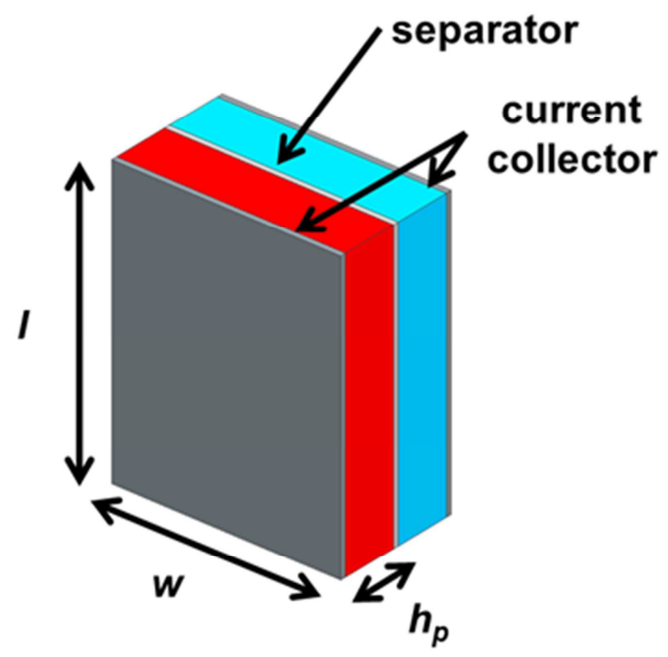

Fig. 2. Dimensions of single cell. The variables $l$ and $w$ are dimensions of the current collector and separator. The variable $h_{p}$ is the electrode thickness or distance from the current collector to the separator. 

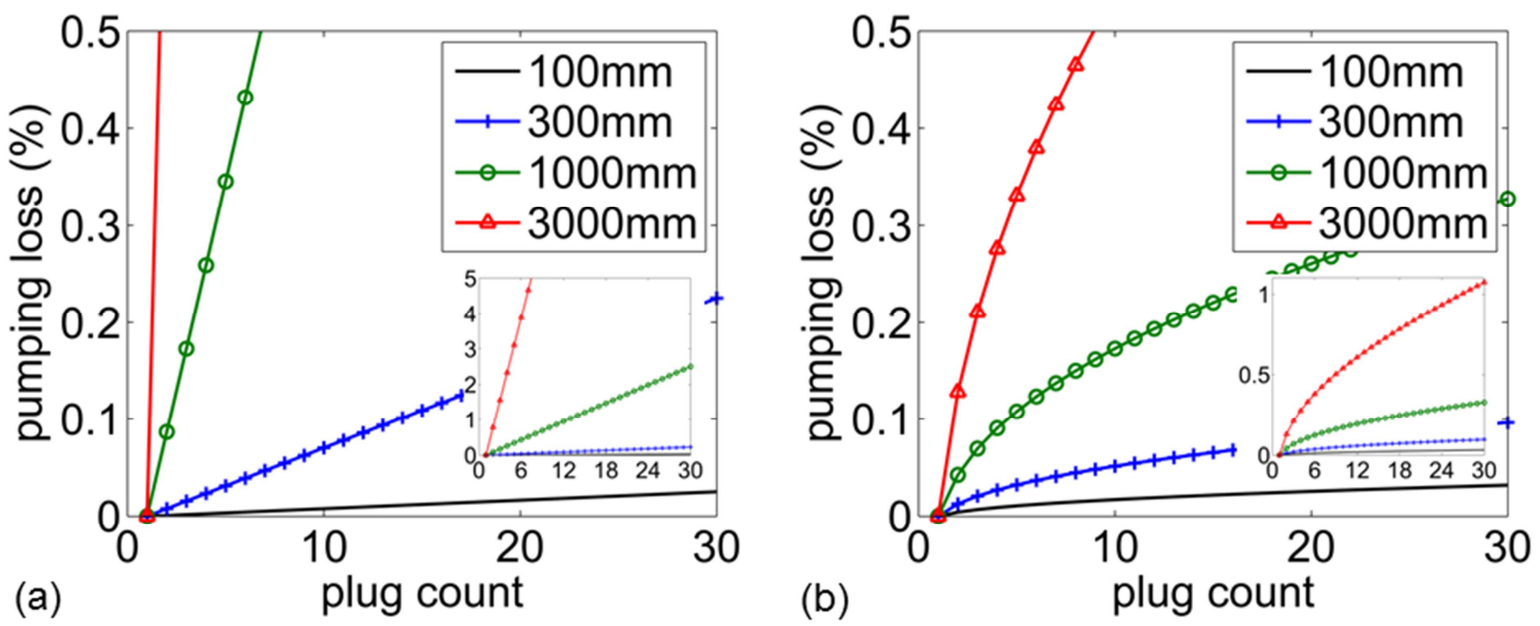

Fig. 3. Losses in efficiency due to pumping for an electrode thickness that yields $95 \%$ energy efficiency including electrochemical and mechanical energy losses for (a) vanadium-redox solution and (b) 10 vol\% LFP/15 vol\% LTP suspension cells cycled at C/5 system-level rate and with membrane length $l$ listed in the legend.
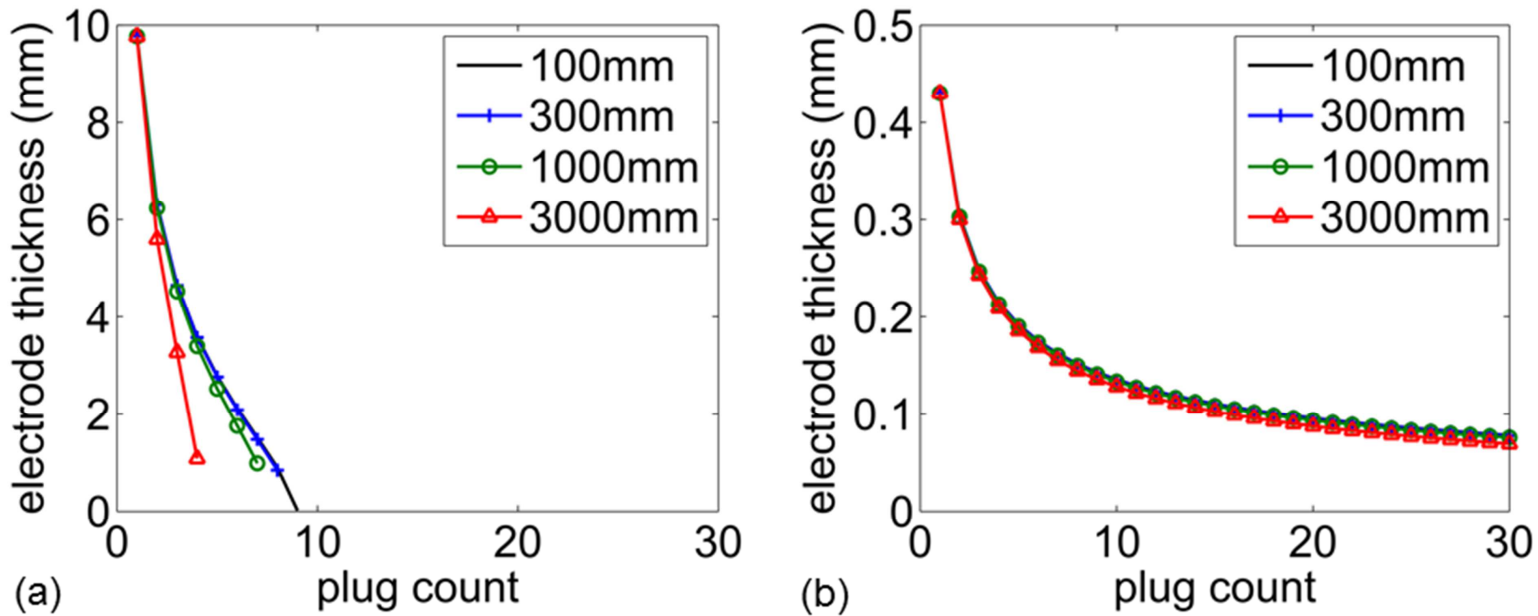

Fig. 4. Electrode thickness $h_{p}$ corresponding to $95 \%$ energy efficiency including electrochemical and mechanical energy losses for (a) vanadium-redox solution and (b) 10 vol\% LFP/15 vol\% LTP suspension cells cycled at C/5 system-level rate and with separator length $l$ listed in the legend. 

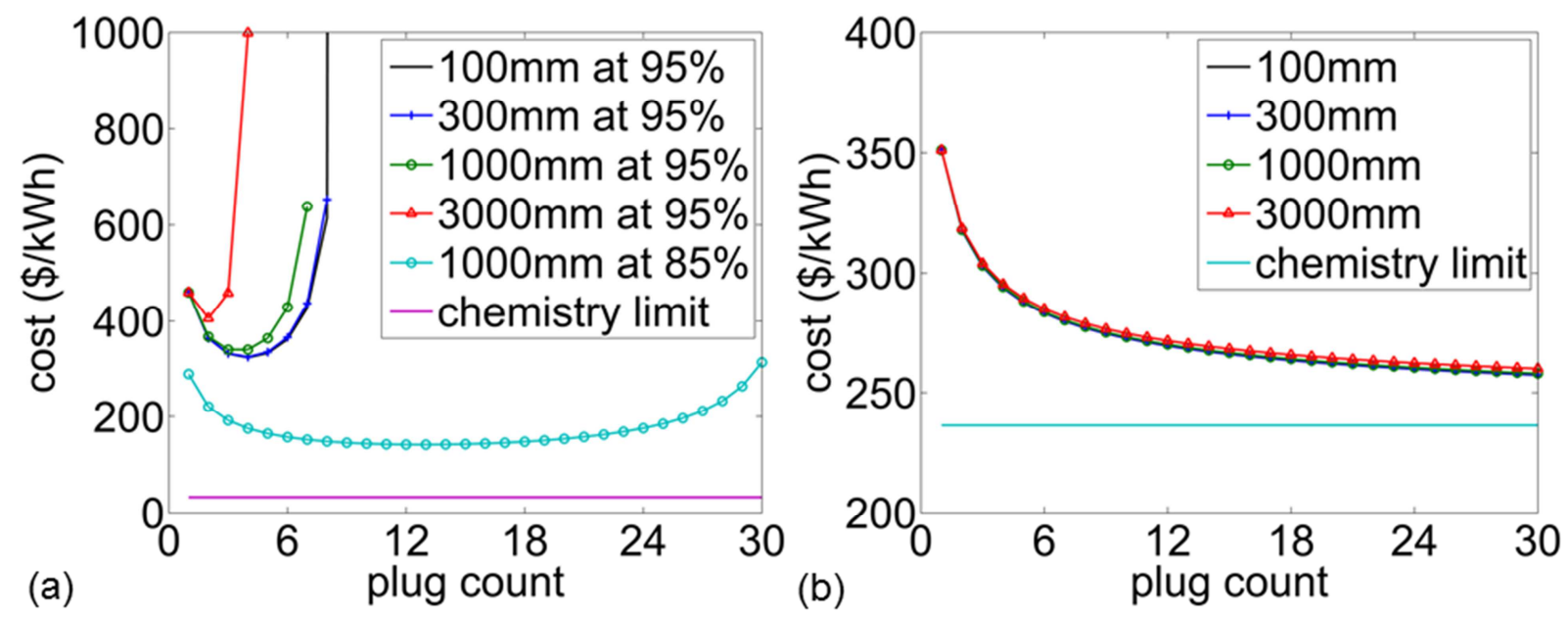

Fig. 5. Stack cost corresponding to $95 \%$ energy efficiency including electrochemical and mechanical energy losses for (a) vanadium-redox solution and (b) 10 vol\% LFP/15 vol\% LTP suspension cells cycled at $\mathrm{C} / 5$ system-level rate and with separator length $l$ listed in the legend. In (a), results corresponding to $85 \%$ round trip energy efficiency for $1000 \mathrm{~mm}$ separator length are also shown. 

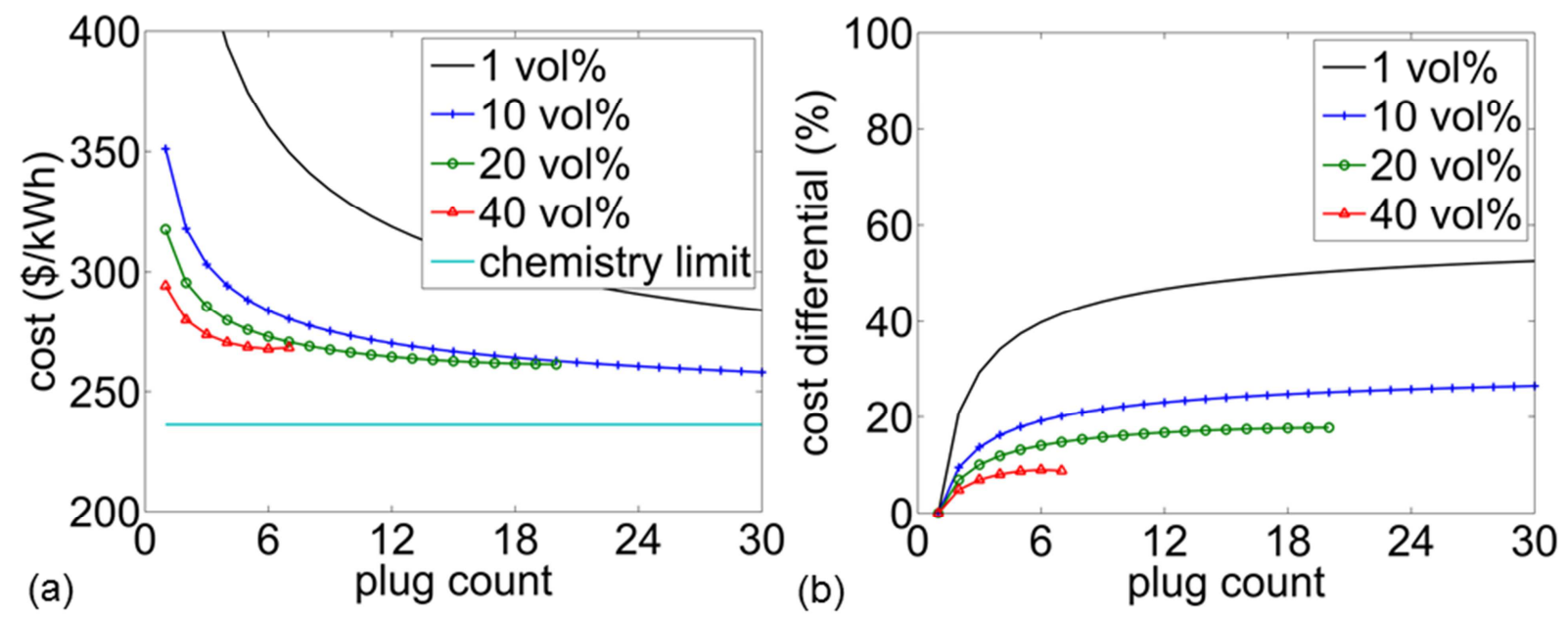

Fig. 6. (a) Stack cost corresponding to 95\% energy efficiency including electrochemical and mechanical energy losses for LFP/LTP suspensions with volume percentages listed in the legend with separator lengths of $1 \mathrm{~m}$. (b) (Cost of static cell - cost of flow cell)/(cost of static cell)*100 for LFP/LTP suspensions with the same volume percentages and separator length as in (a). 